\title{
Some generalized Gronwall-Bellman type nonlinear delay integral inequalities for discontinuous functions
}

\section{Bin Zheng ${ }^{*}$}

\section{"Correspondence:}

zhengbin2601@126.com

School of Science, Shandong

University of Technology, Zibo,

Shandong 255049, China

\begin{abstract}
In this work, some new generalized Gronwall-Bellman type nonlinear delay integral inequalities for discontinuous functions are established, which can be used as a handy tool in the quantitative and qualitative analysis of solutions of certain integral equations. The established results generalize the main results of Gao and Meng in (Math. Pract. Theory 39(22):198-203, 2009).
\end{abstract}

MSC: 26D10; 26D15

Keywords: delay integral inequality; discontinuous function; integral equation; bounded

\section{Introduction}

In recent years, the research of various mathematical inequalities has been paid much attention to by many authors, and many integral inequalities have been established, which provide handy tools for investigating the quantitative and qualitative properties of solutions of integral and differential equations. Among these inequalities, Gronwall-Bellman type integral inequalities are of particular importance as such inequalities provide explicit bounds for unknown functions, and a lot of generalizations of Gronwall-Bellman type integral inequalities have been established (for example, see [1-16] and the references therein). But to our knowledge, most of the known integral inequalities are concerned with continuous functions, while very few authors undertake research in integral inequalities for discontinuous functions [14-16]. Furthermore, delay integral inequalities containing integration on infinite intervals for discontinuous functions have not been reported in the literature so far.

In [1], Gao and Meng established a generalized Gronwall-Bellman type integral inequality, named Mate-Nevai type nonlinear integral inequality for continuous functions, which is one case of inequalities containing integration on infinite intervals for continuous functions. The related theorem reads as follows.

Theorem A Suppose that $u \in C\left(\mathbb{R}_{+}, \mathbb{R}_{+}\right), H, F \in C\left(\mathbb{R}_{+} \times \mathbb{R}_{+}, \mathbb{R}_{+}\right)$, and $H(t, s), F(t, s)$ are decreasing in $t$ for every fixed $s . \phi \in C\left(\mathbb{R}_{+}, \mathbb{R}_{+}\right)$is strictly increasing, and $\phi(\infty)=\infty$. $\psi \in C\left(\mathbb{R}_{+}, \mathbb{R}_{+}\right)$is increasing. $\psi\left(\phi^{-1}\right) \in C\left(\mathbb{R}_{+}, \mathbb{R}_{+}\right)$is strictly increasing, and $\psi\left(\phi^{-1}\right)$ is sub- 
multiplicative. If for $C \geq 0$ and $t \in \mathbb{R}_{+}, u(t)$ satisfies

$$
\phi(u(t)) \leq C+\int_{t}^{\infty} H(t, s) \phi(u(s)) d s+\int_{t}^{\infty} F(t, s) \psi(u(s)) d s,
$$

then for $0 \leq T \leq t \leq \infty$, we have

$$
\begin{aligned}
u(t) \leq & \phi^{-1}\left\{\exp \left[\int_{t}^{\infty} H(t, s) d s\right] G^{-1}[G(C)\right. \\
& \left.\left.+\int_{t}^{\infty} F(t, s) \psi\left(\phi^{-1}\left(\exp \left(\int_{t}^{\infty} H(t, s) d \xi\right)\right)\right) d s\right]\right\}
\end{aligned}
$$

where $G(z)=\int_{z_{0}}^{z} \frac{1}{\psi\left(\phi^{-1}(s)\right)} d s, z \geq z_{0}>0, G^{-1}$ is the inverse of $G$, and $T \in \mathbb{R}_{+}$is chosen so that

$$
G(C)+\int_{t}^{\infty} F(t, s) \psi\left(\phi^{-1}\left(\exp \left(\int_{t}^{\infty} H(t, s) d \xi\right)\right)\right) d s \in \operatorname{Dom}\left(G^{-1}\right), \quad T \leq t<\infty .
$$

The inequality above appears to be useful in deriving bounds of solutions of certain integral equations, but it is inadequate to deal with boundedness for solutions of both integral equations for discontinuous functions and delay integral equations.

In the present paper, motivated by the work above, we establish some new generalized Mate-Nevai type nonlinear delay integral inequalities for discontinuous functions, which extend the main results in [1]. The establishment for the desired inequalities will be discussed in 1-D and 2-D cases respectively.

\section{Main results}

In the rest of the paper, we denote the set of real numbers as $\mathbb{R}$, and $\mathbb{R}_{+}=[0, \infty)$.

Theorem 2.1 Suppose that $u(t)$ is a nonnegative continuous function defined on $\mathbb{R}_{+}$with the first kind of discontinuities at the points $t_{i}, i=1,2, \ldots, n$, and $0 \leq t_{1}<t_{2}<\cdots<t_{n}<t_{n+1}=$ $\infty . f, g \in C\left(\mathbb{R}_{+} \times \mathbb{R}_{+}, \mathbb{R}_{+}\right), f_{2}^{\prime}, g_{2}^{\prime} \in C\left(\mathbb{R}_{+} \times \mathbb{R}_{+}, \mathbb{R}\right)$, and $f(s, t), g(s, t)$ are decreasing in $t$ for every fixed s. $p>0$ is a constant. $\tau \in C\left(\mathbb{R}_{+}, \mathbb{R}_{+}\right)$with $\tau(t) \leq t$, and $\tau(t) \geq t_{i}$ for $\forall t \in\left[t_{i}, t_{i+1}\right)$, $i=0,1, \ldots, n . \omega \in C\left(\mathbb{R}_{+}, \mathbb{R}_{+}\right)$, and $\omega$ is nondecreasing with $\omega(u)>0$ for $u>0$. Furthermore, $\omega$ is submultiplicative, that is, $\omega(\alpha \gamma) \leq \omega(\alpha) \omega(\gamma)$ for $\forall \alpha, \gamma \in \mathbb{R}_{+}$. $C, \beta_{i}$ are constants, and $C \geq 0, \beta_{i}>0, i=1,2, \ldots, n$. If for $t \in \mathbb{R}_{+}, u(t)$ satisfies the following inequality:

$$
u(t) \leq C+\int_{t}^{\infty} f(s, t) u(\tau(s)) d s+\int_{t}^{\infty} g(s, t) \omega(u(\tau(s))) d s+\sum_{t<t_{j}<\infty} \beta_{j} u\left(t_{j}-0\right),
$$

then

$$
\begin{aligned}
u(t) \leq & G_{i-1}^{-1}\left[-\int_{t}^{t_{i}} \widetilde{g}_{i-1}(s) \exp \left(-\widetilde{f}_{i-1}(s)\right) \omega\left(\exp \left(\widetilde{f}_{i-1}(s)\right)\right) d s\right] \exp \left(\widetilde{f}_{i-1}(t)\right), \\
& t \in\left[t_{i-1}, t_{i}\right), i=1,2, \ldots, n+1
\end{aligned}
$$


where

$$
\left\{\begin{array}{l}
G_{i-1}(z)=\int_{b_{i-1}}^{z} \frac{1}{\omega(s)} d s, \quad i=1,2, \ldots, n+1, \\
b_{n}=C, \quad b_{i-1}=a_{i}+\beta_{i} a_{i}, \quad i=1,2, \ldots n, \\
a_{i}=G_{i}^{-1}\left[-\int_{t_{i}}^{t_{i+1}} \widetilde{g}_{i}(s) \exp \left(-\widetilde{f}_{i}(s)\right) \omega\left(\exp \left(\widetilde{f}_{i}(s)\right)\right) d s\right] \exp \left(\widetilde{f}_{i}\left(t_{i}\right)\right), \quad i=1,2, \ldots, n, \\
\widetilde{f}_{i-1}(t)=\int_{t}^{t_{i}} f(s, t) d s, \quad i=1,2, \ldots, n+1, \\
\widetilde{g}_{i-1}(t)=\frac{d\left(\int_{t}^{t_{i}} g(s, t) d s\right)}{d t}, \quad i=1,2, \ldots, n+1 .
\end{array}\right.
$$

Proof Denote the right-hand side of (2.1) by $v(t)$. Then

$$
u(t) \leq v(t) .
$$

Define

$$
\widetilde{v}_{i}(t)=b_{i}+\int_{t}^{\infty} f(s, t) u(\tau(s)) d s+\int_{t}^{\infty} g(s, t) \omega(u(\tau(s))) d s, \quad i=0,1, \ldots, n .
$$

Case 1: If $t \in\left[t_{n}, \infty\right)$ (in fact, $t_{n+1}=\infty$ ), then $v(t)=\widetilde{v}_{n}(t)$. From the definition of $\tau$, we have $\tau(t) \geq t_{n}$ for $t \in\left[t_{n}, \infty\right)$. So, $\tau(t) \in\left[t_{n}, \infty\right)$, and from (2.4) we have

$$
u(\tau(t)) \leq \widetilde{v}_{n}(\tau(t)) \leq \widetilde{v}_{n}(t)
$$

Then

$$
\widetilde{v}_{n}(t) \leq C+\int_{t}^{\infty} f(s, t) \widetilde{v}_{n}(s) d s+\int_{t}^{\infty} g(s, t) \omega\left(\widetilde{v}_{n}(s)\right) d s
$$

and

$$
\begin{aligned}
\widetilde{v}_{n}^{\prime}(t)= & -f(t, t) u(\tau(t))+\int_{t}^{\infty} \frac{\partial f(s, t)}{\partial t} u(\tau(s)) d s-g(t, t) \omega(u(\tau(t))) \\
& +\int_{t}^{\infty} \frac{\partial g(s, t)}{\partial t} \omega(u(\tau(s))) d s \\
\geq & -f(t, t) \widetilde{v}_{n}(t)+\int_{t}^{\infty} \frac{\partial f(s, t)}{\partial t} \widetilde{v}_{n}(s) d s-g(t, t) \omega\left(\widetilde{v}_{n}(t)\right)+\int_{t}^{\infty} \frac{\partial g(s, t)}{\partial t} \omega\left(\widetilde{v}_{n}(s)\right) d s \\
\geq & {\left[-f(t, t)+\int_{t}^{\infty} \frac{\partial f(s, t)}{\partial t} d s\right] \widetilde{v}_{n}(t)+\left[-g(t, t)+\int_{t}^{\infty} \frac{\partial g(s, t)}{\partial t} d s\right] \omega\left(\widetilde{v}_{n}(t)\right) } \\
= & \frac{d\left(\int_{t}^{\infty} f(s, t) d s\right)}{d t} \widetilde{v}_{n}(t)+\frac{d\left(\int_{t}^{\infty} g(s, t) d s\right)}{d t} \omega\left(\widetilde{v}_{n}(t)\right) \\
= & \frac{d \widetilde{f}_{n}(t)}{d t} \widetilde{v}_{n}(t)+\widetilde{g}_{n}(t) \omega\left(\widetilde{v}_{n}(s)\right),
\end{aligned}
$$

where $\widetilde{f}_{n}, \widetilde{g}_{n}$ are defined in (2.3), and obviously $\widetilde{g}_{n}(t) \leq 0$. Furthermore, we have

$$
\widetilde{v}_{n}^{\prime}(t)-\frac{d \widetilde{f}_{n}(t)}{d t} \widetilde{v}_{n}(t) \geq \widetilde{g}_{n}(t) \omega\left(\widetilde{v}_{n}(t)\right)
$$


Multiplying $\exp \left(\widetilde{f}_{n}(t)\right)$ on both sides of (2.8) yields

$$
\left[\widetilde{v}_{n}(t) \exp \left(-\widetilde{f}_{n}(t)\right)\right]^{\prime} \geq \widetilde{g}_{n}(t) \exp \left(-\widetilde{f}_{n}(t)\right) \omega\left(\widetilde{v}_{n}(t)\right)
$$

Setting $t=s$ in (2.9), by $\widetilde{v}_{n}(\infty)=C=b_{n}$, an integration for (2.9) with respect to $s$ from $t$ to $\infty$ yields

$$
b_{n}-\widetilde{v}_{n}(t) \exp \left(-\widetilde{f}_{n}(t)\right) \geq \int_{t}^{\infty} \widetilde{g}_{n}(s) \exp \left(-\widetilde{f}_{n}(s)\right) \omega\left(\widetilde{v}_{n}(s)\right) d s,
$$

which implies

$$
\widetilde{v}_{n}(t) \leq\left\{b_{n}-\int_{t}^{\infty} \widetilde{g}_{n}(s) \exp \left(-\widetilde{f}_{n}(s)\right) \omega\left(\widetilde{v}_{n}(s)\right) d s\right\} \exp \left(\widetilde{f}_{n}(t)\right) .
$$

Define $c(t)=b_{n}-\int_{t}^{\infty} \widetilde{g}_{n}(s) \exp \left(-\widetilde{f}_{n}(s)\right) \omega\left(\widetilde{v}_{n}(s)\right) d s$. Then

$$
\widetilde{v}_{n}(t) \leq c(t) \exp \left(\widetilde{f}_{n}(t)\right)
$$

Since $\widetilde{g}_{n}(t) \leq 0$, and $\omega$ is submultiplicative, we have

$$
\begin{aligned}
c^{\prime}(t) & =\widetilde{g}_{n}(t) \exp \left(-\widetilde{f}_{n}(t)\right) \omega\left(\widetilde{v}_{n}(t)\right) \\
& \geq \widetilde{g}_{n}(t) \exp \left(-\widetilde{f}_{n}(t)\right) \omega\left(c(t) \exp \left(\widetilde{f}_{n}(t)\right)\right) \\
& \geq \widetilde{g}_{n}(t) \exp \left(-\widetilde{f}_{n}(t)\right) \omega(c(t)) \omega\left(\exp \left(\widetilde{f}_{n}(t)\right)\right),
\end{aligned}
$$

that is,

$$
\frac{c^{\prime}(t)}{\omega(c(t))} \geq \widetilde{g}_{n}(t) \exp \left(-\widetilde{f}_{n}(t)\right) \omega\left(\exp \left(\widetilde{f}_{n}(t)\right)\right) .
$$

Setting $t=s$ in (2.13), an integration for (2.13) with respect to $s$ from $t$ to $\infty$ yields

$$
G_{n}(c(\infty))-G_{n}(c(t)) \geq \int_{t}^{\infty} \widetilde{g}_{n}(s) \exp \left(-\widetilde{f}_{n}(s)\right) \omega\left(\exp \left(\widetilde{f}_{n}(s)\right)\right) d s .
$$

By $c(\infty)=b_{n}, G_{n}\left(b_{n}\right)=0$, and $G_{n}$ is increasing, we obtain

$$
c(t) \leq G_{n}^{-1}\left[-\int_{t}^{\infty} \tilde{g}_{n}(s) \exp \left(-\widetilde{f}_{n}(s)\right) \omega\left(\exp \left(\widetilde{f}_{n}(s)\right)\right) d s\right] .
$$

Combining (2.12) and (2.14), we obtain

$$
u(t) \leq \widetilde{v}_{n}(t) \leq G_{n}^{-1}\left[-\int_{t}^{\infty} \widetilde{g}_{n}(s) \exp \left(-\widetilde{f}_{n}(s)\right) \omega\left(\exp \left(\widetilde{f}_{n}(s)\right)\right) d s\right] \exp \left(\widetilde{f}_{n}(t)\right) .
$$

Especially,

$$
u\left(t_{n}\right) \leq \widetilde{v}_{n}\left(t_{n}\right) \leq G_{n}^{-1}\left[-\int_{t_{n}}^{\infty} \widetilde{g}_{n}(s) \exp \left(-\widetilde{f}_{n}(s)\right) \omega\left(\exp \left(\widetilde{f}_{n}(s)\right)\right) d s\right] \exp \left(\widetilde{f}_{n}\left(t_{n}\right)\right)=a_{n},
$$


where $a_{n}$ is defined in (2.3). At the same time, we have

$$
u\left(t_{n}-0\right) \leq \widetilde{v}_{n}\left(t_{n}-0\right) \leq a_{n}
$$

Case 2: If $t \in\left[t_{n-1}, t_{n}\right)$, then from (2.1), (2.16) and (2.17), we have

$$
\begin{aligned}
u(t) \leq & C+\int_{t}^{\infty} f(s, t) u(\tau(s)) d s+\int_{t}^{\infty} g(s, t) \omega(u(\tau(s))) d s+\beta_{n} u\left(t_{n}-0\right) \\
= & b_{n}+\int_{t_{n}}^{\infty} f(s, t) u(\tau(s)) d s+\int_{t_{n}}^{\infty} g(s, t) \omega(u(\tau(s))) d s \\
& +\int_{t}^{t_{n}} f(s, t) u(\tau(s)) d s+\int_{t}^{t_{n}} g(s, t) \omega(u(\tau(s))) d s+\beta_{n} u\left(t_{n}-0\right) \\
\leq & a_{n}+\beta_{n} a_{n}+\int_{t}^{t_{n}} f(s, t) u(\tau(s)) d s+\int_{t}^{t_{n}} g(s, t) \omega(u(\tau(s))) d s \\
= & b_{n-1}+\int_{t}^{t_{n}} f(s, t) u(\tau(s)) d s+\int_{t}^{t_{n}} g(s, t) \omega(u(\tau(s))) d s=\widetilde{v}_{n-1}(t) .
\end{aligned}
$$

Then, following in a similar manner as in Case 1, we obtain

$$
\begin{aligned}
u(t) & \leq \widetilde{v}_{n-1}(t) \\
& \left.\leq G_{n-1}^{-1}\left[-\int_{t}^{t_{n}} \widetilde{g}_{n-1}(s) \exp \left(-\widetilde{f}_{n-1}(s)\right) \omega\left(\exp \widetilde{f}_{n-1}(s)\right)\right) d s\right] \exp \left(\widetilde{f}_{n-1}(t)\right) .
\end{aligned}
$$

Especially,

$$
\left\{\begin{aligned}
u\left(t_{n-1}\right) & \leq \widetilde{v}_{n-1}\left(t_{n-1}\right) \\
& \leq G_{n-1}^{-1}\left[-\int_{t_{n-1}}^{t_{n}} \widetilde{g}_{n-1}(s) \exp \left(-\widetilde{f}_{n-1}(s)\right) \omega\left(\exp \left(\widetilde{f}_{n-1}(s)\right)\right) d s\right] \exp \left(\widetilde{f}_{n-1}\left(t_{n-1}\right)\right)=a_{n-1}, \\
u\left(t_{n-1}-0\right) & \leq \widetilde{v}_{n-1}\left(t_{n_{1}}-0\right) \leq a_{n-1}
\end{aligned}\right.
$$

where $a_{n-1}$ is defined in (2.3).

Case 3: If for $t \in\left[t_{j}, t_{j+1}\right), j=i, i+1, \ldots, n$, the following inequalities hold:

$$
\left\{\begin{array}{l}
u(t) \leq \widetilde{v}_{j}(t) \leq G_{j}^{-1}\left[-\int_{t}^{t_{j+1}} \widetilde{g}_{j}(s) \exp \left(-\widetilde{f}_{j}(s)\right) \omega\left(\exp \left(\tilde{f}_{j}(s)\right)\right) d s\right] \exp \left(\tilde{f}_{j}(t)\right), \\
u\left(t_{j}\right) \leq \widetilde{v}_{j}\left(t_{j}\right) \leq G_{j}^{-1}\left[-\int_{t_{j}}^{t_{j+1}} \widetilde{g}_{j}(s) \exp \left(-\widetilde{f}_{j}(s)\right) \omega\left(\exp \left(\widetilde{f}_{j}(s)\right)\right) d s\right] \exp \left(\widetilde{f}_{j}\left(t_{j}\right)\right)=a_{j}, \\
u\left(t_{j}-0\right) \leq \widetilde{v}_{j}\left(t_{j}-0\right) \leq a_{j},
\end{array}\right.
$$

where $a_{j}$ is defined in (2.3). Then for $t \in\left[t_{i-1}, t_{i}\right)$, from (2.1) we obtain

$$
\begin{aligned}
u(t) \leq & C+\int_{t}^{\infty} f(s, t) u(\tau(s)) d s+\int_{t}^{\infty} g(s, t) \omega(u(\tau(s))) d s+\sum_{t<t_{j}<\infty} \beta_{j} u\left(t_{j}-0\right) \\
= & C+\int_{t_{i}}^{\infty} f(s, t) u(\tau(s)) d s+\int_{t_{i}}^{\infty} g(s, t) \omega(u(\tau(s))) d s \\
& +\int_{t}^{t_{i}} f(s, t) u(\tau(s)) d s+\int_{t}^{t_{i}} g(s, t) \omega(u(\tau(s))) d s+\sum_{t<t_{j}<\infty} \beta_{j} u\left(t_{j}-0\right)
\end{aligned}
$$




$$
\begin{aligned}
& \leq a_{i}+\beta_{i} a_{i}+\int_{t}^{t_{i}} f(s, t) u(\tau(s)) d s+\int_{t}^{t_{i}} g(s, t) \omega(u(\tau(s))) d s \\
& =b_{i-1}+\int_{t}^{t_{i}} f(s, t) u(\tau(s)) d s+\int_{t}^{t_{i}} g(s, t) \omega(u(\tau(s))) d s=\widetilde{v}_{i-1}(t) .
\end{aligned}
$$

Then, following in a similar manner as in Case 1, we obtain

$$
u(t) \leq \widetilde{v}_{i-1}(t) \leq G_{i-1}^{-1}\left[-\int_{t}^{t_{i}} \widetilde{g}_{i-1}(s) \exp \left(-\widetilde{f}_{i-1}(s)\right) \omega\left(\exp \left(\widetilde{f}_{i-1}(s)\right)\right) d s\right] \exp \left(\widetilde{f}_{i-1}(t)\right)
$$

and the proof is complete.

Remark 2.1 If we take $u(t)=\phi(\widetilde{u}(t)), \omega=\psi \phi^{-1}, \tau(t)=t$, and furthermore $u(t)$ is continuous on $\mathbb{R}_{+}$, then Theorem 2.1 reduces to Theorem A (i.e., [1, Theorem 1]).

Remark 2.2 Under the assumptions of Remark 2.1, furthermore, if we take $\phi(\widetilde{u}(t))=\widetilde{u}^{p}(t)$, then Theorem 2.1 reduces to [1, Corollary 1$]$.

Remark 2.3 If we take $\omega(u(t))=u^{q}(t)$ in Theorem 2.1, Remark 2.1 and Remark 2.2, then we can obtain three corollaries, which are omitted here.

Based on Theorem 2.1, we will establish another Mate-Nevai type inequality for discontinuous functions in the 2-D case.

Theorem 2.2 Suppose $u(x, y)$ is a nonnegative continuous function on $\Omega=\bigcup_{i, j \geq 1} \Omega_{i, j}, \Omega_{i, j}=$ $\left\{(x, y) \mid x_{i-1} \leq x<x_{i}, y_{j-1} \leq y<y_{j}\right\}$ with the exception at the points $\left(x_{i}, y_{i}\right), i=1,2, \ldots, n$, where there are finite jumps, and $0 \leq x_{0}<x_{1}<\cdots<x_{n}<x_{n+1}=\infty, 0 \leq y_{0}<y_{1}<\cdots<y_{n}<y_{n+1}=$ $\infty . f, g \in C\left(\mathbb{R}_{+} \times \mathbb{R}_{+}, \mathbb{R}_{+}\right) . \tau_{1}(x) \in C\left(\mathbb{R}_{+}, \mathbb{R}_{+}\right)$with $\tau_{1}(x) \leq x$, and $\tau_{1}(x) \geq x_{i}$ for $\forall x \in\left[x_{i}, x_{i+1}\right)$, $i=0,1, \ldots, n . \tau_{2}(y) \in C\left(\mathbb{R}_{+}, \mathbb{R}_{+}\right)$with $\tau_{2}(y) \leq y$, and $\tau(y) \geq y_{i}$ for $\forall y \in\left[y_{i}, y_{i+1}\right), i=0,1, \ldots, n$. $\omega$ is defined the same as in Theorem 2.1. Furthermore, $f(x, y)=0, g(x, y)=0$ for $(x, y) \in \Omega_{i, j}$, $i \neq j . C>0$ is a constant. If for $(x, y) \in \Omega, u(x, y)$ satisfies the following inequality:

$$
\begin{aligned}
u(x, y) \leq & C+\int_{x}^{\infty} \int_{y}^{\infty} f(s, t) u\left(\tau_{1}(s), \tau_{2}(t)\right) d t d s+\int_{x}^{\infty} \int_{y}^{\infty} g(s, t) \omega\left(u\left(\tau_{1}(s), \tau_{2}(t)\right)\right) d t d s \\
& +\sum_{x<x_{j}<\infty, y<y_{j}<\infty} \beta_{j} u\left(x_{j}-0, y_{j}-0\right),
\end{aligned}
$$

then

$$
\begin{gathered}
u(x, y) \leq \widetilde{G}_{i-1}^{-1}\left[-\int_{x}^{x_{i}} \widetilde{g}_{i-1}(s, y) \exp \left(-\widetilde{f}_{i-1}(s, y)\right) \omega\left(\exp \left(\widetilde{f}_{i-1}(s, y)\right)\right) d s\right] \exp \left(\widetilde{f}_{i-1}(x, y)\right), \\
(x, y) \in \Omega_{i, i}, i=1,2, \ldots, n+1
\end{gathered}
$$


where

$$
\left\{\begin{array}{l}
\widetilde{G}_{i-1}(z)=\int_{\tilde{b}_{i-1}}^{z} \frac{1}{\omega(s)} d s, \quad i=1,2, \ldots, n+1, \\
\widetilde{b}_{n}=C, \quad \widetilde{b}_{i-1}=\widetilde{a}_{i}+\beta_{i} \widetilde{a}_{i}, \quad i=1,2, \ldots, n, \\
\widetilde{a}_{i}=\widetilde{G}_{i}^{-1}\left[-\int_{x_{i}}^{x_{i+1}} \widetilde{g}_{i}\left(s, y_{i}\right) \exp \left(-\widetilde{f}_{i}\left(s, y_{i}\right)\right) \omega\left(\exp \left(\widetilde{f}_{i}\left(s, y_{i}\right)\right)\right) d s\right] \exp \left(\widetilde{f}_{i}\left(x_{i}, y_{i}\right)\right) \\
\quad i=1,2, \ldots, n \\
\widetilde{f}_{i-1}(x, y)=\int_{x}^{x_{i}} \int_{y}^{y_{i}} f(s, t) d t d s, \quad i=1,2, \ldots, n+1 \\
\widetilde{g}_{i-1}(x, y)=-\int_{y}^{y_{i}} g(x, t) d t, \quad i=1,2, \ldots, n+1 .
\end{array}\right.
$$

Proof Denote the right-hand side of $(2.23)$ by $v(x, y)$. Then

$$
u(x, y) \leq v(x, y) .
$$

Define

$$
\begin{gathered}
\widetilde{v}_{i}(x, y)=b_{i}+\int_{x}^{\infty} \int_{y}^{\infty} f(s, t) u\left(\tau_{1}(s), \tau_{2}(t)\right) d t d s+\int_{t}^{\infty} g(s, t) \omega\left(u\left(\tau_{1}(s), \tau_{2}(t)\right)\right) d t d s, \\
i=0,1, \ldots, n .
\end{gathered}
$$

Case 1: If $(x, y) \in \Omega_{n+1, n+1}$, then $v(x, y)=\widetilde{v}_{n}(x, y)$. As $\tau_{1}(x) \geq x_{n}$ for $x \in\left[x_{n}, \infty\right)$ and $\tau_{2}(y) \geq$ $y_{n}$ for $y \in\left[y_{n}, \infty\right)$, so $\left(\tau_{1}(x), \tau_{2}(y)\right) \in \Omega_{n+1, n+1}$, and from (2.26) we have

$$
u\left(\tau_{1}(x), \tau_{2}(y)\right) \leq \widetilde{v}_{n}\left(\tau_{1}(x), \tau_{2}(y)\right) \leq \widetilde{v}_{n}(x, y) .
$$

Then

$$
\widetilde{v}_{n}(x, y) \leq C+\int_{x}^{\infty} \int_{y}^{\infty} f(s, t) \widetilde{v}_{n}(s, t) d t d s+\int_{x}^{\infty} \int_{y}^{\infty} g(s, t) \omega\left(\widetilde{v}_{n}(s, t)\right) d t d s
$$

and

$$
\begin{aligned}
{\left[\widetilde{v}_{n}(x, y)\right]_{x} } & \geq-\left[\int_{y}^{\infty} f(x, t) \widetilde{v}_{n}(x, t) d t+\int_{y}^{\infty} g(x, t) \omega\left(\widetilde{v}_{n}(x, t)\right) d t\right] \\
& \geq-\left[\int_{y}^{\infty} f(x, t) d t\right] \widetilde{v}_{n}(x, y)-\left[\int_{y}^{\infty} g(x, t) d t\right] \omega\left(\widetilde{v}_{n}(x, y)\right) \\
& =\left[\widetilde{f}_{n}(x, y)\right]_{x} \widetilde{v}_{n}(x, y)+\widetilde{g}_{n}(x, y) \omega\left(\widetilde{v}_{n}(x, y)\right),
\end{aligned}
$$

where $\widetilde{f}_{n}, \widetilde{g}_{n}$ are defined in (2.25), and obviously $\widetilde{g}_{n}(x, y) \leq 0$.

Furthermore, we have

$$
\left[\widetilde{v}_{n}(x, y)\right]_{x}-\left[\widetilde{f}_{n}(x, y)\right]_{x} \widetilde{v}_{n}(x, y) \geq \widetilde{g}_{n}(x, y) \omega\left(\widetilde{v}_{n}(x, y)\right) .
$$

Multiplying $\exp \left(\widetilde{f}_{n}(x, y)\right)$ on both sides of $(2.30)$ yields

$$
\left[\widetilde{v}_{n}(x, y) \exp \left(-\widetilde{f}_{n}(x, y)\right)\right]_{x} \geq \widetilde{g}_{n}(x, y) \exp \left(-\widetilde{f}_{n}(x, y)\right) \omega\left(\widetilde{v}_{n}(x, y)\right) .
$$


Setting $x=s$ in (2.31), by $\widetilde{v}_{n}(\infty, y)=C=\widetilde{b}_{n}$, an integration for (2.31) with respect to $s$ from $x$ to $\infty$ yields

$$
\widetilde{b}_{n}-\widetilde{v}_{n}(x, y) \exp \left(-\widetilde{f}_{n}(x, y)\right) \geq \int_{x}^{\infty} \widetilde{g}_{n}(s, y) \exp \left(-\widetilde{f}_{n}(s, y)\right) \omega\left(\widetilde{v}_{n}(s, y)\right) d s,
$$

which implies

$$
\widetilde{v}_{n}(x, y) \leq\left\{\widetilde{b}_{n}-\int_{x}^{\infty} \widetilde{g}_{n}(s, y) \exp \left(-\widetilde{f}_{n}(s, y)\right) \omega\left(\widetilde{v}_{n}(s, y)\right) d s\right\} \exp \left(\widetilde{f}_{n}(x, y)\right) .
$$

Denote $c(x, y)$ by $\widetilde{b}_{n}-\int_{x}^{\infty} \widetilde{g}_{n}(s, y) \exp \left(-\widetilde{f}_{n}(s, y)\right) \omega\left(\widetilde{v}_{n}(s, y)\right) d s$. Then

$$
\widetilde{v}_{n}(x, y) \leq c(x, y) \exp \left(\widetilde{f}_{n}(x, y)\right)
$$

By $\widetilde{g}_{n}(x, y) \leq 0$, we have

$$
\begin{aligned}
{[c(x, y)]_{x} } & =\widetilde{g}_{n}(x, y) \exp \left(-\widetilde{f}_{n}(x, y)\right) \omega\left(\widetilde{v}_{n}(x, y)\right) \\
& \geq \widetilde{g}_{n}(x, y) \exp \left(-\widetilde{f}_{n}(x, y)\right) \omega\left(c(x, y) \exp \left(\widetilde{f}_{n}(x, y)\right)\right) \\
& \geq \widetilde{g}_{n}(x, y) \exp \left(-\widetilde{f}_{n}(x, y)\right) \omega(c(x, y)) \omega\left(\exp \left(\widetilde{f}_{n}(x, y)\right)\right),
\end{aligned}
$$

that is,

$$
\frac{[c(x, y)]_{x}}{\omega(c(x, y))} \geq \widetilde{g}_{n}(x, y) \exp \left(-\widetilde{f}_{n}(x, y)\right) \omega\left(\exp \left(\widetilde{f}_{n}(x, y)\right)\right) .
$$

Setting $x=s$ in (2.35), and an integration for (2.35) with respect to $s$ from $x$ to $\infty$ yields

$$
\widetilde{G}_{n}(c(\infty, y))-\widetilde{G}_{n}(c(x, y)) \geq \int_{x}^{\infty} \widetilde{g}_{n}(s, y) \exp \left(-\widetilde{f}_{n}(s, y)\right) \omega\left(\exp \left(\widetilde{f}_{n}(s, y)\right)\right) d s .
$$

Since $c(\infty, y)=\widetilde{b}_{n}, \widetilde{G}_{n}\left(\widetilde{b}_{n}\right)=0$, and $\widetilde{G}_{n}$ is increasing, it follows that

$$
c(x, y) \leq \widetilde{G}_{n}^{-1}\left[-\int_{x}^{\infty} \widetilde{g}_{n}(s, y) \exp \left(-\widetilde{f}_{n}(s, y)\right) \omega\left(\exp \left(\widetilde{f}_{n}(s, y)\right)\right) d s\right] .
$$

Combining (2.34) and (2.36), we obtain

$$
\begin{aligned}
u(x, y) & \leq \widetilde{v}_{n}(x, y) \\
& \leq G_{n}^{-1}\left[-\int_{x}^{\infty} \widetilde{g}_{n}(s, y) \exp \left(-\widetilde{f}_{n}(s, y)\right) \omega\left(\exp \left(\widetilde{f}_{n}(s, y)\right)\right) d s\right] \exp \left(\widetilde{f}_{n}(x, y)\right) .
\end{aligned}
$$

Especially,

$$
\begin{aligned}
u\left(x_{n}, y_{n}\right) \leq & \widetilde{v}_{n}\left(x_{n}, y_{n}\right) \leq G_{n}^{-1}\left[-\int_{x_{n}}^{\infty} \widetilde{g}_{n}\left(s, y_{n}\right) \exp \left(-\widetilde{f}_{n}\left(s, y_{n}\right)\right) \omega\left(\exp \left(\widetilde{f}_{n}\left(s, y_{n}\right)\right)\right) d s\right] \\
& \times \exp \left(\widetilde{f}_{n}\left(x_{n}, y_{n}\right)\right)=\widetilde{a}_{n},
\end{aligned}
$$


where $\widetilde{a}_{n}$ is defined in (2.25). At the same time, we have

$$
u\left(x_{n}-0, y_{n}-0\right) \leq \widetilde{v}_{n}\left(x_{n}-0, y_{n}-0\right) \leq \widetilde{a}_{n} .
$$

Case 2: If $(x, y) \in \Omega_{n, n}$, then from (2.23), (2.38) and (2.39), under the given conditions $f(x, y)=0, g(x, y)=0$ for $(x, y) \in \Omega_{i, j}, i \neq j$, we have

$$
\begin{aligned}
u(x, y) \leq & C+\int_{x}^{\infty} \int_{y}^{\infty} f(s, t) u\left(\tau_{1}(s), \tau_{2}(t)\right) d t d s+\int_{x}^{\infty} \int_{y}^{\infty} g(s, t) \omega\left(u\left(\tau_{1}(s), \tau_{2}(t)\right)\right) d t d s \\
& +\beta_{n} u\left(x_{n}-0, y_{n}-0\right), \\
= & \widetilde{b}_{n}+\int_{x_{n}}^{\infty} \int_{y_{n}}^{\infty} f(s, t) u\left(\tau_{1}(s), \tau_{2}(t)\right) d s+\int_{x_{n}}^{\infty} \int_{y_{n}}^{\infty} g(s, t) \omega\left(u\left(\tau_{1}(s), \tau_{2}(t)\right)\right) d s \\
& +\int_{x}^{x_{n}} \int_{y}^{y_{n}} f(s, t) u\left(\tau_{1}(s), \tau_{2}(t)\right) d s+\int_{x}^{x_{n}} \int_{y}^{y_{n}} g(s, t) \omega\left(u\left(\tau_{1}(s), \tau_{2}(t)\right)\right) d s \\
& +\beta_{n} u\left(x_{n}-0, y_{n}-0\right) \\
\leq & \tilde{a}_{n}+\beta_{n} \tilde{a}_{n}+\int_{x}^{x_{n}} \int_{y}^{y_{n}} f(s, t) u\left(\tau_{1}(s), \tau_{2}(t)\right) d s \\
& +\int_{x}^{x_{n}} \int_{y}^{y_{n}} g(s, t) \omega\left(u\left(\tau_{1}(s), \tau_{2}(t)\right)\right) d s \\
= & \widetilde{b}_{n-1}+\int_{x}^{x_{n}} \int_{y}^{y_{n}} f(s, t) u\left(\tau_{1}(s), \tau_{2}(t)\right) d s+\int_{x}^{x_{n}} \int_{y}^{y_{n}} g(s, t) \omega\left(u\left(\tau_{1}(s), \tau_{2}(t)\right)\right) d s \\
= & \widetilde{v}_{n-1}(x, y) .
\end{aligned}
$$

Then, following in a similar manner as in Case 1, we obtain

$$
\begin{aligned}
u(x, y) \leq & \widetilde{v}_{n-1}(x, y) \leq \widetilde{G}_{n-1}^{-1}\left[-\int_{x}^{x_{n}} \widetilde{g}_{n-1}(s, y) \exp \left(-\widetilde{f}_{n-1}(s, y)\right) \omega\left(\exp \left(\widetilde{f}_{n-1}(s, y)\right)\right) d s\right] \\
& \times \exp \left(\widetilde{f}_{n-1}(x, y)\right) .
\end{aligned}
$$

Especially,

$$
\left\{\begin{array}{l}
u\left(x_{n-1}, y_{n-1}\right) \leq \widetilde{v}_{n-1}\left(x_{n-1}, y_{n-1}\right) \\
\quad \leq \widetilde{G}_{n-1}^{-1}\left[-\int_{x_{n-1}}^{x_{n}} \widetilde{g}_{n-1}(s, y) \exp \left(-\widetilde{f}_{n-1}(s, y)\right) \omega\left(\exp \left(\widetilde{f}_{n-1}(s, y)\right)\right) d s\right] \\
\quad \times \exp \left(\widetilde{f}_{n-1}\left(x_{n-1}, y_{n-1}\right)\right)=\widetilde{a}_{n-1}, \\
u\left(x_{n-1}-0, y_{n-1}-0\right) \leq \widetilde{v}_{n-1}\left(x_{n-1}-0, y_{n-1}-0\right) \leq \widetilde{a}_{n-1},
\end{array}\right.
$$

where $\widetilde{a}_{n-1}$ is defined in (2.25).

Case 3: If for $(x, y) \in \Omega_{j, j}, j=i+1, \ldots, n$, the following inequalities hold:

$$
\left\{\begin{array}{l}
u(x, y) \leq \widetilde{v}_{j}(x, y) \\
\leq G_{j}^{-1}\left[-\int_{x}^{t_{j+1}} \widetilde{g}_{j}(s, y) \exp \left(-\widetilde{f}_{j}(s, y)\right) \omega\left(\exp \left(\widetilde{f}_{j}(s, y)\right)\right) d s\right] \exp \left(\widetilde{f}_{j}(x, y)\right), \\
u\left(x_{j}, y_{j}\right) \leq \widetilde{v}_{j}\left(x_{j}, y_{j}\right) \leq G_{j}^{-1}\left[-\int_{x_{j}}^{x_{j+1}} \widetilde{g}_{j}(s, y) \exp \left(-\widetilde{f}_{j}(s, y)\right) \omega\left(\exp \left(\widetilde{f}_{j}(s, y)\right)\right) d s\right] \\
\quad \quad \times \exp \left(\widetilde{f}_{j}\left(x_{j}, y_{j}\right)\right)=\widetilde{a}_{j}, \\
u\left(x_{j}-0, y_{j}-0\right) \leq \widetilde{v}_{j}\left(x_{j}-0, y_{j}-0\right) \leq \widetilde{a}_{j},
\end{array}\right.
$$


where $\tilde{a}_{j}$ is defined in (2.25), then for $(x, y) \in \Omega_{i, i}$, by (2.23), we obtain

$$
\begin{aligned}
u(x, y) \leq & C+\int_{x}^{\infty} \int_{y}^{\infty} f(s, t) u\left(\tau_{1}(s), \tau_{2}(t)\right) d t d s+\int_{x}^{\infty} \int_{y}^{\infty} g(s, t) \omega\left(u\left(\tau_{1}(s), \tau_{2}(t)\right)\right) d t d s \\
& +\sum_{x<x_{j}<\infty, y<y_{j}<\infty} \beta_{j} u\left(x_{j}-0, y_{j}-0\right) \\
= & C+\int_{x_{i}}^{\infty} \int_{y_{i}}^{\infty} f(s, t) u\left(\tau_{1}(s), \tau_{2}(t)\right) d t d s+\int_{x_{i}}^{\infty} \int_{y_{i}}^{\infty} g(s, t) \omega\left(u\left(\tau_{1}(s), \tau_{2}(t)\right)\right) d t d s \\
& +\int_{x}^{x_{i}} \int_{y}^{y_{i}} f(s, t) u\left(\tau_{1}(s), \tau_{2}(t)\right) d t d s+\int_{x}^{x_{i}} \int_{y}^{y_{i}} g(s, t) \omega\left(u\left(\tau_{1}(s), \tau_{2}(t)\right)\right) d t d s \\
& +\sum_{x<x_{j}<\infty, y<y_{j}<\infty} \beta_{j} u\left(x_{j}-0, y_{j}-0\right) \\
\leq & \widetilde{a}_{i}+\beta_{i} \widetilde{a}_{i}+\int_{x}^{x_{i}} \int_{y}^{y_{i}} f(s, t) u\left(\tau_{1}(s), \tau_{2}(t)\right) d t d s \\
& +\int_{x}^{x_{i}} \int_{y}^{y_{i}} g(s, t) \omega\left(u\left(\tau_{1}(s), \tau_{2}(t)\right)\right) d t d s \\
= & \widetilde{b}_{i-1}+\int_{x}^{x_{i}} \int_{y}^{y_{i}} f(s, t) u\left(\tau_{1}(s), \tau_{2}(t)\right) d t d s+\int_{x}^{x_{i}} \int_{y}^{y_{i}} g(s, t) \omega\left(u\left(\tau_{1}(s), \tau_{2}(t)\right)\right) d t d s \\
= & \widetilde{v}_{i-1}(x, y) .
\end{aligned}
$$

Then, following in a similar manner as in Case 1, we obtain

$$
\begin{aligned}
u(x, y) \leq & \widetilde{v}_{i-1}(x, y) \leq \widetilde{G}_{i-1}^{-1}\left[-\int_{x}^{t_{i}} \widetilde{g}_{i-1}(s, y) \exp \left(-\widetilde{f}_{i-1}(s, y)\right) \omega\left(\exp \left(\widetilde{f}_{i-1}(s, y)\right)\right) d s\right] \\
& \times \exp \left(\widetilde{f}_{i-1}(x, y)\right)
\end{aligned}
$$

and the proof is complete.

Remark 2.4 If we take $u(x, y)=\phi(\widetilde{u}(x, y))$ and, furthermore, $\phi(u(x, y))=u^{q}(x, y)$, or take $\omega(u)=u^{q}$ in Theorem 2.2, then we can obtain corresponding corollaries, which are omitted here.

\section{Application}

In this section, we present one application to illustrate the validity of our results in deriving explicit bounds for the discontinuous solutions of certain integral equations.

Consider an integral equation of the form

$$
u(t)=u(\infty)+\int_{t}^{\infty} M(s, t, u(\tau(s))) d s+\sum_{t<t_{j}<\infty} \beta_{j} u\left(t_{j}-0\right), \quad \forall t \in \mathbb{R}_{+},
$$

where $u$ is a continuous function defined on $\mathbb{R}_{+}$with the first kind of discontinuities at the points $t_{i}, i=1,2, \ldots, n$, and $0 \leq t_{0}<t_{1}<t_{2}<\cdots<t_{n}<t_{n+1}=\infty . \tau \in C\left(\mathbb{R}_{+}, \mathbb{R}_{+}\right)$with $\tau(t) \leq t$, and $\tau(t) \geq t_{i}$ for $\forall t \in\left[t_{i}, t_{i+1}\right), i=0,1, \ldots, n . \beta_{j} \geq 0, j=1,2, \ldots, n$, and $M \in C\left(\mathbb{R}_{+}^{2} \times \mathbb{R}, \mathbb{R}\right)$. 
Theorem 3.1 Assume that $u(t)$ is a solution of Eq. (3.1), and

$$
\left\{\begin{array}{l}
|u(\infty)| \leq C, \\
|M(s, t, u)| \leq f(s, t)|u|+g(s, t)|u|^{q}, \\
\omega(z)=z^{q}, \quad z \in \mathbb{R}_{+},
\end{array}\right.
$$

where $q, C$ are constants with $0<q<1, C \geq 0, f, g \in C\left(\mathbb{R}_{+} \times \mathbb{R}_{+}, \mathbb{R}_{+}\right), f_{2}^{\prime}, g_{2}^{\prime} \in C\left(\mathbb{R}_{+} \times \mathbb{R}_{+}, \mathbb{R}\right)$, and $f(s, t), g(s, t)$ are decreasing in $t$ for every fixed $s$. Then we have

$$
\begin{aligned}
|u(t)| \leq & G_{i-1}^{-1}\left[-\int_{t}^{t_{i}} \widetilde{g}_{i-1}(s) \exp \left(-\widetilde{f}_{i-1}(s)\right) \omega\left(\exp \left(\widetilde{f}_{i-1}(s)\right)\right) d s\right] \exp \left(\widetilde{f}_{i-1}(t)\right), \\
& t \in\left[t_{i-1}, t_{i}\right), i=1,2, \ldots, n+1
\end{aligned}
$$

where $G_{i}, b_{i}, a_{i}, \widetilde{f}_{i}, \widetilde{g}_{i}$ are defined the same as in (2.3), and $t_{n+1}=\infty$.

Proof From (3.1) we have

$$
\begin{aligned}
|u(t)| & \leq|u(\infty)|+\int_{t}^{\infty}|M(s, t, u(\tau(s)))| d s+\sum_{t<t_{j}<\infty} \beta_{j}\left|u\left(t_{j}-0\right)\right| \\
& \leq C+\int_{t}^{\infty}\left[f(s, t)|u(\tau(s))|+g(s, t)|u(\tau(s))|^{q}\right] d s+\sum_{t<t_{j}<\infty} \beta_{j}\left|u\left(t_{j}-0\right)\right| \\
& =C+\int_{t}^{\infty}[f(s, t)|u(\tau(s))|+g(s, t) \omega(|u(\tau(s))|)] d s+\sum_{t<j_{j}<\infty} \beta_{j}\left|u\left(t_{j}-0\right)\right| .
\end{aligned}
$$

Then a suitable application of Theorem 2.1 yields the desired result.

Theorem 3.2 Under the conditions of Theorem 3.1, furthermore, we have

$$
\begin{aligned}
|u(t)| \leq & \left\{(1-q)\left[-\int_{t}^{t_{i}} \widetilde{g}_{i-1}(s) \exp \left(-\widetilde{f}_{i-1}(s)\right) \exp \left(\widetilde{q}_{i-1}(s)\right) d s\right]+b_{i-1}^{1-q}\right\}^{\frac{1}{1-q}} \\
& \times \exp \left(\widetilde{f}_{i-1}(t)\right) .
\end{aligned}
$$

Proof As long as we notice $\omega(z)=z^{q}$, and

$$
G_{i-1}(z)=\int_{b_{i-1}}^{z} \frac{1}{\omega(s)} d s=\int_{b_{i-1}}^{z} \frac{1}{s^{q}} d s=\frac{1}{1-q}\left(z^{1-q}-b_{i-1}^{1-q}\right), \quad i=1,2, \ldots, n+1,
$$

combining (3.5) and Theorem 3.1, we can easily deduce the desired result.

\section{Competing interests}

The author declares that they have no competing interests.

\section{Acknowledgements}

The authors would like to thank the referees very much for their careful comments and valuable suggestions on this paper.

Received: 7 November 2012 Accepted: 30 May 2013 Published: 21 June 2013 


\section{References}

1. Gao, QL, Meng, FW: On some new Mate-Nevai inequalities. Math. Pract. Theory 39(22), 198-203 (2009)

2. Li, WN, Han, MA, Meng, FW: Some new delay integral inequalities and their applications. J. Comput. Appl. Math. 180 $191-200(2005)$

3. Lipovan, O: A retarded integral inequality and its applications. J. Math. Anal. Appl. 285, 436-443 (2003)

4. $\mathrm{Ma}, \mathrm{QH}$, Yang, EH: Some new Gronwall-Bellman-Bihari type integral inequalities with delay. Period. Math. Hung. 44(2), 225-238 (2002)

5. Yuan, ZL, Yuan, XW, Meng, FW: Some new delay integral inequalities and their applications. Appl. Math. Comput. 208, 231-237 (2009)

6. Lipovan, O: Integral inequalities for retarded Volterra equations. J. Math. Anal. Appl. 322, 349-358 (2006)

7. Pachpatte, BG: Explicit bounds on certain integral inequalities. J. Math. Anal. Appl. 267, 48-61 (2002)

8. Pachpatte, BG: On some new nonlinear retarded integral inequalities. J. Inequal. Pure Appl. Math. 5, Article ID 80 (2004)

9. Sun, YG: On retarded integral inequalities and their applications. J. Math. Anal. Appl. 301, 265-275 (2005)

10. Ferreira, RAC, Torres, DFM: Generalized retarded integral inequalities. Appl. Math. Lett. 22, 876-881 (2009)

11. Xu, R, Sun, YG: On retarded integral inequalities in two independent variables and their applications. Appl. Math. Comput. 182, 1260-1266 (2006)

12. Jiang, FC, Meng, FW: Explicit bounds on some new nonlinear integral inequality with delay. J. Comput. Appl. Math. 205, 479-486 (2007)

13. Li, LZ, Meng, FW, He, LL: Some generalized integral inequalities and their applications. J. Math. Anal. Appl. 372 339-349 (2010)

14. Gallo, A, Piccirillo, AM: About some new generalizations of Bellman-Bihari results for integro-functional inequalities with discontinuous functions and applications. Nonlinear Anal. 71, e2276-e2287 (2009)

15. Iovane, G: Some new integral inequalities of Bellman-Bihari type with delay for discontinuous functions. Nonlinear Anal. 66, 498-508 (2007)

16. Borysenko, S, lovane, G: About some new integral inequalities of Wendroff type for discontinuous functions. Nonlinear Anal. 66, 2190-2203 (2007)

doi:10.1186/1029-242X-2013-297

Cite this article as: Zheng: Some generalized Gronwall-Bellman type nonlinear delay integral inequalities for discontinuous functions. Journal of Inequalities and Applications 2013 2013:297.

\section{Submit your manuscript to a SpringerOpen ${ }^{\circ}$ journal and benefit from:}

- Convenient online submission

- Rigorous peer review

- Immediate publication on acceptance

Open access: articles freely available online

- High visibility within the field

- Retaining the copyright to your article 